(C) 2002 International Press

Adv. Theor. Math. Phys. 6 (2002) 619-642

\title{
Geometric Transitions
}

\section{and Open String Instantons}

\author{
D.-E. Diaconescu, ${ }^{1}$ B. Florea, ${ }^{2}$ and A. Grassi ${ }^{3}$ \\ ${ }^{1}$ Department of Physics and Astronomy, Rutgers University, \\ Piscataway, NJ 08855-0849, USA \\ email: duiliu@physics.rutgers.edu \\ ${ }^{2}$ Mathematical Institute, University of Oxford, \\ 24-29 St. Giles', Oxford OX1 3LB, England \\ email: florea@maths.ox.ac.uk \\ ${ }^{3}$ Department of Mathematics, University of Pennsylvania, \\ Philadelphia, PA 19104-6395, USA \\ email: grassi@math.upenn.edu
}

We investigate the physical and mathematical structure of a new class of geometric transitions proposed by Aganagic and Vafa. The distinctive aspect of these transitions is the presence of open string instanton corrections to Chern-Simons theory. We find a precise match between open and closed string topological amplitudes applying a beautiful idea proposed by Witten some time ago. The closed string amplitudes are reproduced from an open string perspective as a result of a fascinating interplay of enumerative techniques and Chern-Simons computations.

e-print archive: http://xxx.lanl.gov/hep-th/0205234 


\section{Introduction}

Open topological strings on Calabi-Yau manifolds have been the subject of much recent activity in the context of geometric transitions and enumerative geometry [1]-[6],[14]-[19],[20]-[23],[25]-[28],[30]-[35],[37]-[39]. In particular, Aganagic and Vafa [5] found a new class of large $N$ geometric dualities which yield very interesting predictions for open topological string amplitudes on noncompact Calabi-Yau threefolds. Recall that the original geometric transition discovered by Gopakumar and Vafa [14] relates open strings on a deformed conifold to closed strings on the blow-up of the same conifold singularity. As shown by Witten many years ago [40], the topological open string on a deformed conifold is equivalent to $U(N)$ Chern-Simons theory on the vanishing $S^{3}$ cycle. The new transitions of [5] predict a similar relation for a more complex geometric set-up in which the open string theory is corrected by instanton effects. Such situations have been anticipated by Witten in [40], where the instanton corrections have been elegantly interpreted as non-local Wilson loop operators in the Chern-Simons action. The Wilson loops in question are boundaries of holomorphic discs (or higher genus bordered Riemann surfaces) interpreted as knots in $S^{3}$. Then the computation of the open string free energy reduces to a fascinating combination of open string enumerative geometry and perturbative Chern-Simons theory.

In this paper we show that these techniques can be successfully applied to the large $N$ duality proposed in [5], resulting in a precise match between open and closed string amplitudes. The computation of open string amplitudes in this background entails two different aspects. One has first to compute the instanton corrections to the Chern-Simons effective action, which is essentially a problem in open string enumerative geometry $[16,23,30]$. In fact, a detailed (and quite involved) analysis shows that in this particular model all these corrections are generated by multicovers of a single rigid disc. The corresponding instanton expansion has been predicted by Ooguri and Vafa in [35] and computed explicitly by Katz and Liu [23] and Li and Song [30] . The next step is a Chern-Simons computation, in which the instanton corrections are treated as non-local Wilson loop perturbations [40]. We show that the final result is in perfect agreement with the closed string free energy, provided that one takes into account a certain correction to the duality map. A remarkable aspect of this correspondence is that a priori the closed and open string instanton expansions exhibit different multicover contributions. This discrepancy is miraculously accounted for by the perturbative Chern-Simons corrections, so that in the end we obtain a precise match.

The paper is structured as follows. Section two is a brief review of the geometric construction of [5], and the duality predictions. In section three we review and develop the computation of closed string amplitudes of [5], finding a com- 
plete expression for the closed string free energy. Section four is devoted to open string amplitudes and the duality map, and we conclude with a series of technical considerations on open string morphisms in section five.

\section{The Transitions}

The starting point of our discussion is the first model considered in [5], namely a toric noncompact Calabi-Yau threefold $X$ defined by the toric quotient $\left(\mathbb{C}^{5} \backslash F\right) /\left(\mathbb{C}^{*}\right)^{2}$

$$
\begin{array}{rrrrr}
X_{0} & X_{1} & X_{2} & X_{3} & X_{4} \\
1 & 0 & -1 & 1 & -1 \\
-2 & 1 & 0 & 0 & 1
\end{array}
$$

where the disallowed locus is $F=\left\{X_{0}=X_{3}=0\right\} \cup\left\{X_{1}=X_{3}=0\right\} \cup\left\{X_{1}=\right.$ $\left.X_{4}=0\right\}$. This toric quotient can be equivalently described as a symplectic quotient $\mathbb{C}^{5} / / U(1)^{2}$ with the moment maps

$$
\begin{array}{r}
\left|X_{0}\right|^{2}-\left|X_{2}\right|^{2}+\left|X_{3}\right|^{2}-\left|X_{4}\right|^{2}=\operatorname{Re}(s) \\
-2\left|X_{0}\right|^{2}+\left|X_{1}\right|^{2}+\left|X_{4}\right|^{2}=\operatorname{Re}(t)
\end{array}
$$

where $(s, t)$ are complexified Kähler parameters with $\operatorname{Re}(s)>0, \operatorname{Re}(t)>0$. Note that for $t=s=0$, the quotient (2) is a singular variety described in terms of invariant polynomials $x=-X_{2} X_{3}, y=X_{0} X_{1} X_{4}, u=X_{0} X_{3} X_{4}^{2}, v=X_{0} X_{1}^{2} X_{2}$ by

$$
u v+x y^{2}=0 .
$$

This singularity admits several distinct crepant resolutions corresponding to nonzero values of $(\operatorname{Re}(s), \operatorname{Re}(t))$, which parameterize the extended Kähler cone. As usual, each such resolution corresponds to a different triangulation of the toric diagram, and different resolutions are related by flops. The triangulation corresponding to (2) is represented in fig. 1.

Using this description, it easy to see that the exceptional locus consists of two smooth rational curves $C_{s}, C_{t}$ with normal bundles

$$
N_{C_{s} / X}=\mathcal{O}(-1) \oplus \mathcal{O}(-1), \quad N_{C_{t} / X}=\mathcal{O} \oplus \mathcal{O}(-2)
$$

As suggested by the notation, the two curves have volumes $\int_{C_{s}} J=\operatorname{Re}(s), \int_{C_{t}} J=$ $\operatorname{Re}(t)$. Moreover, $C_{s}$ is rigid while $C_{t}$ moves in a one parameter family on $X$. A 


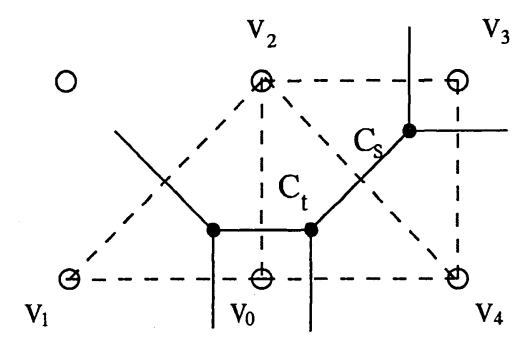

Figure 1: Triangulation of the toric variety (2) with $\operatorname{Re}(s)>0, \operatorname{Re}(t)>0$

very useful and intuitive description of $X$ can be achieved by representing it as a (topological) $T^{2} \times \mathbb{R}$ fibration over $\mathbb{R}^{3}[17,29]$. The discriminant of this fibration is the planar graph represented with continuous lines in fig. 1. In this framework, the curves $C_{s}, C_{t}$ can be described as topological $S^{1}$ fibrations over certain line segments ending on the edges of $\Gamma$. We will not give more details here since this is standard material $[17,29]$. The extremal transition considered in [5] consists of a contraction of $C_{s}$ on $X$, followed by a smoothing of the resulting nodal singularity. Let us denote by $\widehat{X}$ the singular variety obtained in the process. As discussed in [5], $\widehat{X}$ can be realized as a partial resolution of the singularity (3) obtained by blowing-up the plane $u=y=0$

$$
u \rho=y \lambda, \quad u v+x y^{2}=0 .
$$

There are two coordinate patches $U_{1}, U_{2}$ on the total space of the blow-up $(x, y, v, \lambda)$ and $(x, u, v, \rho)$, with transition functions

$$
x=x, \quad v=v, \quad u=y \lambda, \quad \rho=\frac{1}{\lambda} .
$$

This shows that the blow-up $Z$ is isomorphic to the total space of the rank three bundle $\mathcal{O} \oplus \mathcal{O} \oplus \mathcal{O}(-1)$ over $\mathbb{P}^{1}$. The local equations of the proper transform $\widehat{X}$ in the two patches are

$$
v \lambda+x y=0, \quad v+x u \rho^{2}=0 .
$$

Note that there is a conifold singularity left in the first patch, which can be smoothed out by deforming the equations as follows

$$
v \lambda+x y=\mu, \quad v+x u \rho^{2}=\mu \rho
$$

where $\mu \in \Delta^{*}$ is a complex deformation parameter on the unit disc. Throughout this paper we will work at some fixed arbitrary value of $\mu$, which can be 
assumed real and positive without loss of generality ${ }^{1}$. Let $\mathcal{Y} / \Delta$ denote the family parameterized by $\mu$. The generic fiber $Y_{\mu}$ is a smooth noncompact Calabi-Yau threefold, and the central fiber is isomorphic to $\widehat{X}$. Moreover, standard surgery arguments [8] show that the nonzero Betti numbers of a generic smooth fiber are $b_{2}\left(Y_{\mu}\right)=1, b_{3}\left(Y_{\mu}\right)=1$. The third homology is generated by a vanishing 3 -sphere $L_{\mu} \subset Y_{\mu}$, which is the fixed point set of the local antiholomorphic involution $\iota: U_{1} \rightarrow U_{1}, \iota:(x, y, v, \lambda) \rightarrow(\bar{y}, \bar{x}, \bar{\lambda}, \bar{v})$. Note that $L_{\mu}$ is lagrangian with respect to any symplectic form $\omega$ on $Z$ such that $\left.\omega\right|_{U_{1}}$ is odd under the involution $\iota$. Such a symplectic form can be constructed as follows. We can think of $Z$ as a direct product $Z=W \times \mathbb{C}^{2}$, where $W$ is the total space of $\mathcal{O}(-1)$ over $\mathbb{P}^{1}$. We have coordinates $(x, v)$ on the $\mathbb{C}^{2}$ factor, and local coordinates $(y, \lambda)$ and $(u, \rho)$ on $W$. The local antiholomorphic involution $\iota$ is of the form $\iota=\left(\kappa, \kappa^{-1}\right)$, where $\kappa$ is a map $\kappa: \mathbb{C}^{2} \rightarrow U_{1} \cap W, \kappa:(x, v) \rightarrow(\bar{y}, \bar{\lambda})$. Pick a symplectic Kähler form $\eta$ on $W$, and let $\eta_{1}$ be the restriction of $\eta$ to $U_{1} \cap W$. Then $\eta^{\prime}=\kappa^{*} \eta_{1}$ defines a symplectic form on $\mathbb{C}^{2}$, and we can take $\omega=\eta-\eta^{\prime}$ to be the desired symplectic form ${ }^{2}$ on $Z$. From now on we fix such a symplectic form on $Z$. The second homology group $H_{2}\left(Y_{\mu}, L_{\mu} ; \mathbb{Z}\right)=\mathbb{Z}$ is generated by a holomorphic disc $D$ in $Y_{\mu}$ with boundary on $L_{\mu}$, which can be constructed as follows. Let $D$ be the disc $|t| \geq \mu^{1 / 2}$ in a projective plane $\mathbb{P}^{1}$ with homogeneous coordinates $\left[t_{1}, t_{2}\right]$, and affine coordinates $t=t_{1} / t_{2}, t^{\prime}=t_{2} / t_{1}$. In local coordinate patches, the embedding map $f: D \rightarrow Y_{\mu}$ is given by

$$
\begin{aligned}
& U_{1}: \lambda(t)=t, v(t)=\frac{\mu}{t}, x(t)=0, y(t)=0 \\
& U_{2}: \rho\left(t^{\prime}\right)=t^{\prime}, v\left(t^{\prime}\right)=\mu t^{\prime}, x\left(t^{\prime}\right)=0, u\left(t^{\prime}\right)=0 .
\end{aligned}
$$

It is easy to check that this local description of the map is compatible with the transition functions (6) and that the boundary of $D$ is mapped to $L_{\mu}$. Note that $D$ is preserved by the antiholomorphic involution. It will be proven in section five that $D$ is an integral generator and that there are no holomorphic curves on $Y_{\mu}$. Therefore we can define an open string Kähler parameter as the symplectic area of the disc

$$
t_{o p}=\int_{D} f^{*} \omega
$$

A deformation argument to be detailed in section five shows that $t_{o p}=t$ at classical level.

\footnotetext{
${ }^{1}$ If $\mu=|\mu| e^{\imath \phi}$ with $\phi \neq 0$, we can reduce to $\mu^{\prime}=|\mu| \in \mathbb{R}_{+}$by a change of coordinates $x^{\prime}=x e^{\imath \phi}, v^{\prime}=v e^{\imath \phi}$.

${ }^{2}$ To be more precise, let $\pi_{1,2}$ denote the projections from $Z=W \times \mathbb{C}^{2}$ onto the two factors. Then $\omega=\pi_{1}^{*} \eta-\pi_{2}^{*} \eta^{\prime}$.
} 
In the context of geometric transitions, we have to consider an open string topological theory defined by wrapping $N$ D-branes on the sphere $L_{\mu}$. This theory is well defined since $L_{\mu}$ is lagrangian. Then, large $N$ geometric duality [5] predicts a relation between closed string free energy on $X$ and open string free energy on $Y_{\mu}$ of the form

$$
\left.\mathcal{F}^{c l}\left(\widehat{t}, \widehat{s}, g_{s}\right)\right|_{\widehat{s}=i \lambda}=\mathcal{F}^{o p}\left(\widehat{t}_{o p}, \lambda, g_{s}\right)
$$

where $\lambda=N g_{s}$ is the 't Hooft coupling constant for $U(N)$ Chern-Simons theory on $L_{\mu}$. In this formula, $(\widehat{t}, \widehat{s})$ denote closed string flat coordinates, which are related to the classical complexified Kähler parameters $(t, s)$ by the mirror map. For the present model the relation between $(\widehat{t}, \widehat{s})$ and $(t, s)$ has been discussed in [5]. By analogy $\widehat{t}_{o p}$ denotes an open string flat coordinate corresponding to $t_{o p}$ $[2,3,28,33,34]$. To conclude this section, note that the topological A model amplitudes are independent of $\mu$ by standard decoupling arguments, so we will drop the subscript $\mu$ from now on. In order to check the duality predictions we need exact expressions for both terms in (11), which will be worked out in the next sections.

\section{Closed string amplitudes}

In this section we consider closed string A model amplitudes on $X$. The partition function $\mathcal{F}^{c l}\left(t, s, g_{s}\right)$ has been computed in [5] up to terms depending on $t$. The strategy is to first compute the genus zero partition function using local mirror symmetry, and then write down a complete formula by interpreting the answer in terms of BPS invariants $[13,24,31]$. Note that this method is specific to the present model; in general one cannot derive all the higher genus amplitudes from the genus zero expression and BPS constraints. The resulting expression is ${ }^{3}$

$$
\mathcal{F}^{c l}\left(t, s, g_{s}\right)=\sum_{n \geq 1}\left[\frac{e^{-n s}}{n\left(2 \sin \left(n g_{s} / 2\right)^{2}\right)}+\frac{e^{-n(s+t)}}{n\left(2 \sin \left(n g_{s} / 2\right)^{2}\right)}\right]+(t \text {-dependent terms }) \text {. }
$$

Note that throughout this paper we will mainly consider truncated partition functions, i.e. we will drop the typical polynomial terms which occur in the low genus expansion. In order to run a precision test of the duality (11), we need to find the remaining $t$-dependent terms. This can be done using local mirror symmetry by analogy with [5] The local mirror manifold of the A model described in section two

\footnotetext{
${ }^{3}$ From now on we will denote the flat coordinates by $(t, s)$ dropping the symbol ${ }^{\wedge}$. Although this notation is potentially ambiguous, the meaning should be clear from the context. In particular, topological amplitudes will always be written in terms of flat coordinates.
} 
is a hypersurface $W$ in $\mathbb{C}^{2} \times\left(\mathbb{C}^{*}\right)^{2}$ which can be written in terms of flat coordinates as [5]

$$
z w=\left(1-e^{-u}\right)\left(1-e^{u-t}\right)-e^{-v}\left(1-e^{u-t-s}\right)
$$

where $(z, w)$ are coordinates on $\mathbb{C}^{2}$ and $\left(e^{-u}, e^{-v}\right)$ are single valued open string flat coordinates on $\left(\mathbb{C}^{*}\right)^{2}[2,3,15,28,33,34]$. The terms in (12) have been found by computing the first derivative $\partial_{s} F_{0}(t, s)$ of the genus zero partition function as a classical period in the local mirror geometry $[7,18,19]$. We have

$$
\partial_{s} F_{0}(t, s)=\int_{t+s}^{\Lambda} v(u) d u
$$

where

$$
v(u)=\log \left[\frac{1-e^{u-t-s}}{\left(1-e^{-u}\right)\left(1-e^{u-t}\right)}\right] .
$$

The integral is taken along the semi-infinite contour $\gamma_{s}$ represented in fig. 2, which can be thought as the projection of a lagrangian three-cycle onto the $(u, v)$ plane. Since this cycle has infinite volume, we have to introduce an infrared cut-off $\Lambda$. Evaluation of (15) results in

$$
\partial_{s} F_{0}(t, s)=-\sum_{n \geq 1}\left[\frac{e^{-n(s+t)}}{n^{2}}+\frac{e^{-n s}}{n^{2}}\right]
$$

up to polynomial and $\Lambda$-dependent terms. As explained in [5] this suggests the existence of two stable BPS states with charges $\left[C_{s}\right],\left[C_{s}+C_{t}\right] \in H_{2}(X, \mathbb{Z})$. The terms in (12) represent the contributions of these two states to the BPS invariants.

In order to detect eventual $t$-dependent terms, one has to perform a similar computation of $\partial_{t} F_{0}(t, s)$. The relevant contour is $\gamma_{t}$ represented in fig. 2. We have

$$
\partial_{t} F_{0}=\int_{-\infty}^{0} v(u) d u=\int_{0}^{\infty} v(-u) d u
$$

with $v(u)$ given by (15). Since we are mainly interested in truncated amplitudes, it suffices to compute $\partial_{t}^{2} F_{0}(s, t)$ in order to avoid convergence issues. Then we are left with an easy calculation

$$
\partial_{t}^{2} F_{0}(s, t)=\int_{0}^{\infty}\left[\frac{e^{-u-t-s}}{1-e^{-u-t-s}}-\frac{e^{-u-t}}{1-e^{-u-t}}\right] d u=\sum_{n \geq 1}\left[\frac{e^{-n(t+s)}}{n}-\frac{e^{-n t}}{n}\right]
$$




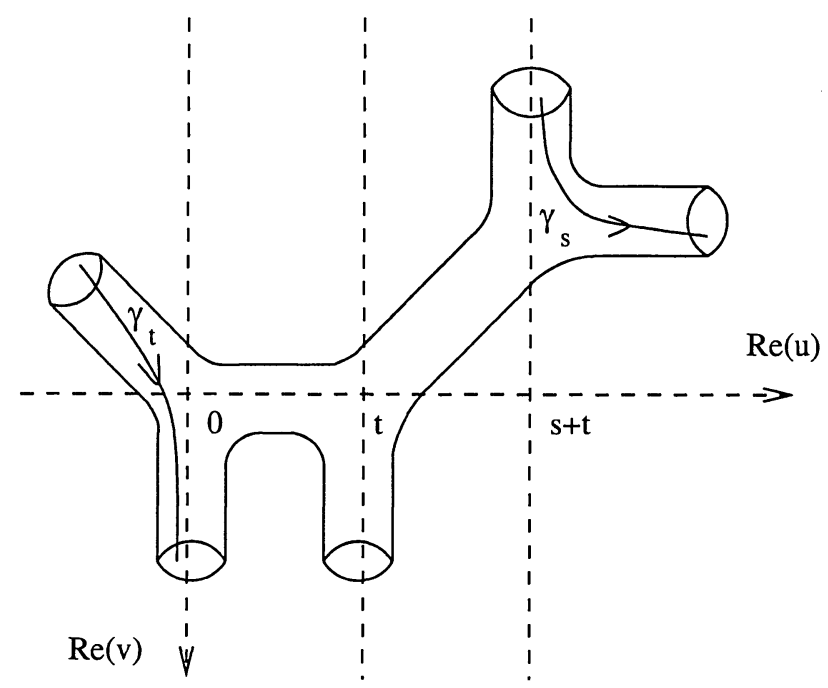

Figure 2: Mirror Riemann surface

Combining (16) and (18) we obtain the following expression for the truncated genus zero amplitude

$$
F_{0}(t, s)=\sum_{n \geq 1}\left[\frac{e^{-n s}}{n^{3}}+\frac{e^{-n(t+s)}}{n^{3}}-\frac{e^{-n t}}{n^{3}}\right] .
$$

Reasoning by analogy with [5], this form of the genus zero amplitude predicts an extra term in the expression of the closed string partition function

$$
\mathcal{F}^{c l}\left(t, s, g_{s}\right)=\sum_{n \geq 1}\left[\frac{e^{-n s}}{n\left(2 \sin \left(n g_{s} / 2\right)^{2}\right)}+\frac{e^{-n(s+t)}}{n\left(2 \sin \left(n g_{s} / 2\right)^{2}\right)}-\frac{e^{-n t}}{n\left(2 \sin \left(n g_{s} / 2\right)^{2}\right)}\right] .
$$

A couple of remarks are in order at this point. The expressions (12), (20) are conjectural at this stage since they have not been confirmed by explicit A model closed string computations. While the terms in (12), have a clear interpretation in terms of BPS invariants, for the third term in (20), such an interpretation is more subtle. The problem is that in the class $\left[C_{t}\right]$ there is a family of rational curves with parameter space $\mathbb{C}$, which is noncompact. Therefore it is not clear how to define the BPS invariants or the Gromov-Witten invariants in this case. A possible approach is to choose an appropriate compactification of $X$ and then take a large volume limit. The resulting invariants will be well defined, but they may be dependent on the particular compactification chosen. This is a drawback, since such compactifications are far from unique. At the same time, we should keep in mind that the above B model computation gives an answer (at least at 
genus zero), without making such a choice. So a legitimate question is whether this computation has an A model counterpart. We will propose below a partial answer, leaving a more conceptual approach for future work.

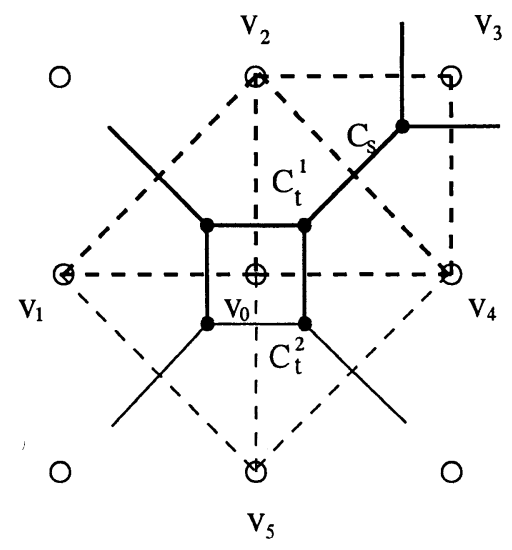

Figure 3: A Toric Compactification of $X$.

To begin with, note that we can compactify this model to a local Calabi-Yau variety containing a Hirzebruch surface $\mathbb{F}_{0}$ and a transverse $(-1,-1)$ curve. This is represented in fig. 3 . The class $\left[C_{t}\right]$ is the fiber class of $\mathbb{F}_{0}$, and $C_{s}$ can be identified with the transverse curve to $\mathbb{F}_{0}$. This is a flopped version of the local $d P_{2}$ model. In this new model, the family of curves in class $\left[C_{t}\right]$ has a compact parameter space $\mathbb{P}^{1}$, and the BPS invariants in the class $d\left[C_{t}\right]$ are very simple [24]. We have $n_{1}^{0}=-e\left(\mathbb{P}^{1}\right)=-2$ and $n_{d}^{r}=0$ for all other values of $(d, r)$, where $e\left(\mathbb{P}^{1}\right)$ is the Euler character of $\mathbb{P}^{1}$. There are two stable BPS states whose wavefunctions are harmonic representatives of $H^{0,0}\left(\mathbb{P}^{1}\right)$ and respectively $H^{1,1}\left(\mathbb{P}^{1}\right)$. In order to recover our original model $X$, we have to take an infinite volume limit of the base $\mathbb{P}^{1}$ of $\mathbb{F}_{0}$. In this limit, the harmonic function in $H^{0,0}\left(\mathbb{P}^{1}\right)$ becomes nonnormalizable, hence the corresponding BPS state is lifted from the spectrum. We are left with a single BPS state corresponding to the harmonic $(1,1)$-form on $\mathbb{P}^{1}$, which can be kept normalizable in this limit. Taking into account the sign as well, we obtain precisely the contribution in (20). To conclude our discussion of closed string amplitudes, note that we can also find an interpretation of (20) in Gromov-Witten theory. Namely, using the same compactified model, one can try to compute the Gromov-Witten invariants in the class $n\left[C_{t}\right]$ by localization with respect to an $\left(\mathbb{C}^{*}\right)^{2}$ action on $\mathbb{F}_{0}[7]$. The $\left(\mathbb{C}^{*}\right)^{2}$ action leaves two fibers invariant, which will be denoted by $C_{t}^{1}, C_{t}^{2} ; C_{t}^{1}$ passes through the intersection point with $C_{s}$, while $C_{t}^{2}$ is a fiber at infinity from the point of view of $X$. The fixed locus of the $\left(\mathbb{C}^{*}\right)^{2}$ action on $\bar{M}_{g}\left(X^{c}, n\left[C_{t}\right]\right)$ consists accordingly of two classes of components corresponding to maps onto $C_{t}^{1}$ and respectively $C_{t}^{2}$. Moreover, by symmetry, it is easy to see that the two classes of components have equal contributions to 
the integral representation of Gromov-Witten invariants for all $(g, n)$. Since the integral in question is taken over a compact moduli space, the sum of the two contributions must be a rational number, hence each individual contribution must also be a rational number. In the decompactification limit, the fiber at infinity is effectively removed, and we are left with a single $\left(\mathbb{C}^{*}\right)^{2}$-invariant component. Therefore we can unambiguously define a closed string expansion on $X$ by simply taking the contribution of a single fixed component of $\bar{M}_{g}\left(X^{c}, n\left[C_{t}\right]\right)$. For genus zero, and low enough degree, it can be checked that this contribution is $-\frac{1}{n^{2}}$ as expected. We conjecture that this procedure also gives the expected answer for all $(g, n)$, but we will not try to prove it here.

To summarize the main point of this section we have presented compelling evidence that the complete closed partition function on $X$ is given by (20). We will show in the next section, that this expression is also in very good agreement with geometric duality predictions.

\section{Open String Amplitudes and The Duality Map}

Let us consider now open string A model amplitudes on the deformation space $Y$ with $N$ D-branes wrapped on $L$. According to [40], the target space effective action for $N$ D-branes wrapping $L$ is $U(N)$ Chern-Simons gauge theory. This theory has played a central role in large $N$ geometric duality for the conifold, starting with [14]. The novelty in the case under consideration is that the Chern-Simons theory is corrected by open string instanton effects. Such situations have been anticipated in section 4.4. of [40], where the instanton effects have been elegantly interpreted as nonlocal Wilson loop corrections to the Chern-Simons action. Suppose for simplicity that we have a single holomorphic disc $D$ in $Y$, with boundary on $L$. Schematically, the full effective action can be written as

$$
S(A)=S_{C S}(A)+F_{\text {inst }}\left(g_{s}, t_{o p}, V\right)
$$

where $t_{o p}$ is an open string flat coordinate ${ }^{4}, g_{s}=\frac{2 \pi}{k+N}$ is the renormalized ChernSimons coupling constant and $V=\operatorname{Pexp} \int_{\Gamma} A$ is the holonomy of the $U(N)$ connection around the boundary $\Gamma$ of $D$, regarded as a knot in $L$. For large Kähler parameters, the instanton corrections can be treated perturbatively from the ChernSimons point of view. Moreover, in the present paper we are interested in a large $N$ 't Hooft expansion so that the open string free energy takes the form

$$
\mathcal{F}_{o p}\left(t_{o p}, \lambda, g_{s}\right)=\mathcal{F}_{o p}^{0}\left(\lambda, g_{s}\right)+\ln \left\langle e^{F_{\text {inst }}\left(g_{s}, t_{o p}, V\right)}\right\rangle,
$$

\footnotetext{
${ }^{4}$ We have dropped again the symbol ${ }^{\wedge}$ in the notation of flat coordinates.
} 
where $\lambda=N g_{s}$ is the 't Hooft coupling constant and $\mathcal{F}_{o p}^{0}\left(\lambda, g_{s}\right)$ is the ChernSimons free energy. In order to evaluate (22) we need an exact expression for the open string instanton corrections $F_{\text {inst }}\left(g_{s}, t_{o p}, V\right)$. We will give here a schematic treatment, leaving some formal details for the next section. Recall that in section two we have constructed a holomorphic disc $D$ embedded in $Y$ with boundary on $L$. We reproduce for convenience the local expression of the embedding map from (9)

$$
\begin{aligned}
& U_{1}: \lambda(t)=t, v(t)=\frac{\mu}{t}, x(t)=0, y(t)=0 \\
& U_{2}: \rho\left(t^{\prime}\right)=t^{\prime}, v\left(t^{\prime}\right)=\mu t^{\prime}, x\left(t^{\prime}\right)=0, u\left(t^{\prime}\right)=0
\end{aligned}
$$

where $D$ is the disc $\left\{|t| \geq \mu^{1 / 2}\right\}=\left\{\left|t^{\prime}\right| \leq \mu^{-1 / 2}\right\}$ in $\mathbb{P}^{1}$ with affine coordinates $\left(t, t^{\prime}\right)$. The boundary of $D$ is mapped to the sphere $L$, which is defined by

$$
\begin{aligned}
& U_{1}: \lambda=\bar{v}, x=\bar{y} \\
& U_{2}: \rho \bar{v}=1, x=\overline{\rho u} .
\end{aligned}
$$

Note that the second coordinate patch $U_{2}$ covers the disc $D$, but it does not cover the entire sphere $L$. In fact, equation (24) shows that $U_{2}$ covers $L$ with the circle $\left\{\lambda=v=0,|x|=|y|=\mu^{1 / 2}\right\}$ removed, since $v, \rho$ are not allowed to vanish on $L \cap U_{2}$. This shows that $L \cap U_{2}$ is diffeomorphic to $S^{1} \times \mathbb{R}^{2}$ and the boundary of $D$ is a section of this cylinder which can be identified with the circle $\left|t^{\prime}\right|=\mu^{-1 / 2}$. Moreover, the normal bundle to $D$ in $Y$ can be identified with a trivial rank two bundle on $D$ with coordinates $(x, u)$ along the fiber. These coordinates are subject to the boundary conditions

$$
x=\overline{\rho u} .
$$

This is a familiar situation since the boundary conditions (25) are identical to the ones of $[23,30]$. In particular, using their results, it follows that the embed$\operatorname{ding} f: D \rightarrow Y$ is rigid. For completeness, note that the boundary conditions (25) define a totally real subbundle $N_{\mathbb{R}}$ of the normal bundle $N$ restricted to the boundary $\Gamma=\partial D$. The pair $\left(N, N_{\mathbb{R}}\right)$ forms a Riemann-Hilbert bundle with generalized Maslov index $\mu\left(N, N_{\mathbb{R}}\right)=-1$. By the double construction, the group of global sections $H^{0}\left(D, \Gamma ; N, N_{\mathbb{R}}\right)$ is zero. We will show in the next section that $D$ is a generator of $H_{2}(Y, L ; \mathbb{Z})=\mathbb{Z}$. By analogy with the closed string situation, the open string instanton numbers should be defined in terms of intersection theory on the moduli space of maps from bordered Riemann surfaces to the pair $(Y, L)$ [23]. The homotopy classes of open string maps $f: \Sigma_{g, h} \rightarrow Y$ with $f\left(\partial \Sigma_{g, h}\right) \subset L$ are classified by the class $\beta=d[D]$, with $d \in \mathbb{Z}$. We denote by $M_{g, h}(Y, L ; d[D])$ the moduli 
space of stable open string maps to the pair $(Y, L)$ such that $f_{*}\left[\Sigma_{g, h}\right]=d[D]$, as defined in [23]. This space should have a suitable compactification $\bar{M}_{g, h}(Y, L ; d[D])$. At the moment, very little is known about the structure of the compactified space $\bar{M}_{g, h}(Y, L ; d[D])$. However, in the particular case considered here, this space has at least certain disconnected components which are familiar from the work of $[23,30]$. The components in question are isomorphic to moduli spaces of multicovers of the disc $D$, which are disconnected components of $\bar{M}_{g, h}(Y, L ; d[D])$ because $D$ is rigid. In addition to the degree $d$, the multicovers of $D$ are also characterized by the winding numbers $n_{\alpha}, \alpha=1, \ldots, h$ of the $h$ boundary components. Therefore we have disconnected components $\bar{M}_{g, h}\left(D, \Gamma ; d, n_{\alpha}\right)$. According to [23] these should be thought of as orbifold spaces with boundary. Note that at this point we do not know if $\bar{M}_{g, h}(Y, L ; d[D])$ has other disconnected components in addition to $\bar{M}_{g, h}\left(D, \Gamma ; d, n_{\alpha}\right)$. The open string Gromov-Witten invariants $N_{d}$ for maps of degree $d$ should be defined in terms of a virtual fundamental class of degree zero on $\bar{M}_{g, h}(Y, L ; d[D])[23]$ whose construction is not known at the present stage. Assuming that such a construction exists, the invariants $N_{d}$ will receive contributions $N_{d, n_{\alpha}}$ from all disconnected components $\bar{M}_{g, h}\left(D, \Gamma ; d, n_{\alpha}\right)$ which have been evaluated in $[23,30]$. In addition, we may have contributions from other disconnected components of $\bar{M}_{g, h}(Y, L ; d[D])$, which are beyond our control at the present stage. We will however present an argument in a later section suggesting that such extra contributions are absent. For the time being, we assume that this is the case, and write down the answer found in $[23,30]$. Since the spaces $\bar{M}_{g, h}\left(D, \Gamma ; d, n_{\alpha}\right)$ have boundary, the invariants $N_{d, n_{\alpha}}$ depend on the choice of boundary conditions, which in the present case amounts to the choice of a framing of $\Gamma[23,30,35]$, i.e. the choice of a homotopy class of sections of $N_{\mathbb{R}}$. In the canonical framing, we have the following instantonq expansion

$$
F_{\text {inst }}\left(g_{s}, t_{o p}, V\right)=i \sum_{d=1}^{\infty} \frac{e^{-d t_{o p}}}{2 d \sin \frac{d g_{s}}{2}} \operatorname{tr} V^{d} .
$$

One can write similar, although more complicated expressions for an arbitrary framing, but we will not give more details here. However, it would be very interesting to understand how duality works at arbitrary framing. We thank Mina Aganagic and Cumrun Vafa for clarifying discussions on this point. The target space effective action (21) becomes

$$
S(A)=S_{C S}(A)+i \sum_{d=1}^{\infty} \frac{e^{-d t_{o p}}}{2 d \sin \frac{d g_{s}}{2}} \operatorname{tr} V^{d} .
$$

Performing a analytic continuation along the lines of $[9,12,14,36]$, the 't Hooft expansion of the Chern-Simons free energy can be set in the form

$$
\mathcal{F}_{o p}^{0}\left(g_{s}, \lambda\right)=\sum_{n=1}^{\infty} \frac{e^{i n \lambda}}{n\left(2 \sin \frac{n g_{s}}{2}\right)^{2}}
$$


where $\lambda=N g_{s}$ is the 't Hooft coupling constant. Recall that throughout this paper we consider truncated string amplitudes, so we have dropped a polynomial and logarithmic piece. Those terms are well understood in the context of geometric duality [14].

In order to finish the computation we have to evaluate the expectation value of $e^{F_{\text {inst }}\left(g_{s}, t_{o p}, V\right)}$. For an unknot with the canonical framing we have [35]

$$
\left\langle\operatorname{tr} V^{k_{1}} \ldots \operatorname{tr} V^{k_{l}}\right\rangle=\left\langle\operatorname{tr} V^{k_{1}}\right\rangle \ldots\left\langle\operatorname{tr} V^{k_{l}}\right\rangle
$$

for any positive integers $k_{1}, \ldots, k_{l}$. Using this property and expanding the exponential, we find

$$
\ln \left\langle e^{F_{2 n s t}\left(g_{s}, t_{o p}, V\right)}\right\rangle=i \sum_{d=1}^{\infty} \frac{e^{-d t_{o p}}}{2 d \sin \frac{d g_{s}}{2}}\left\langle\operatorname{tr} V^{d}\right\rangle .
$$

The expectation values $\left\langle\operatorname{tr} V^{d}\right\rangle$ have been evaluated in [35] for arbitrary $d$, with the result

$$
\left\langle\operatorname{tr} V^{d}\right\rangle=i \frac{e^{-i d \lambda / 2}-e^{i d \lambda / 2}}{2 \sin \frac{d g_{s}}{2}}
$$

Collecting all the results, we obtain the following expression for the open string free energy

$$
\mathcal{F}_{o p}\left(t_{o p}, \lambda, g_{s}\right)=\sum_{n=1}^{\infty} \frac{e^{i n \lambda}}{n\left(2 \sin \frac{n g_{s}}{2}\right)^{2}}+\sum_{d=1}^{\infty} \frac{e^{-d\left(t_{o p}-\frac{2 \lambda}{2}\right)}-e^{-d\left(t_{o p}+\frac{2 \lambda}{2}\right)}}{d\left(2 \sin \frac{d g_{s}}{2}\right)^{2}} .
$$

This expression is to be compared with the closed string free energy worked out in section three.

\subsection{Comparison with Closed String and Duality Map}

Recall that in the previous section we found the closed string free energy on $X$ to be

$$
\mathcal{F}_{c l}\left(g_{s}, s, t\right)=\sum_{n=1}^{\infty} \frac{1}{n\left(2 \sin \frac{n g_{s}}{2}\right)^{2}}\left(e^{-n s}+e^{-n(t+s)}-e^{-n t}\right) .
$$

This formula is remarkably similar to (32), except for the different dependence of the instanton factors on Kähler moduli. However, it is straightforward to see 
that we will obtain a precise agreement if we conjecture a duality map relating the closed and open string Kähler parameters by

$$
s=-i \lambda, \quad t=t_{o p}+\frac{i \lambda}{2} .
$$

Note that the relation $s=-i \lambda$ differs by a sign from the duality map obtained in [13]. Changing the sign in the duality map corresponds to a flop in the closed string geometry. One can check that the alternative duality map $s=i \lambda$ corresponds to a different large radius limit of the model dicussed in section two, namely $\operatorname{Re}(t)>0$, $\operatorname{Re}(s)<0$.

At this point, one may wonder what is the physical interpretation of the half integral shift of the second Kähler parameter. In the large volume regime, $t, t_{o p}$ differ from the classical Kähler parameters by exponentially small corrections. Hence, as discussed in more detail at the end of section five, a classical geometric reasoning would predict a relation of the form $t=t_{o p}$, which is obviously in contradiction with (34).

On the other hand, the shift (34) appears to be a perturbative quantum correction in Chern-Simons theory. Such a correction would not be visible at tree level in the field theory, which is consistent with the treatment of [5]. From a string theory point of view, we can think of this shift as a nonperturbative correction to the open string flat coordinates induced by degenerate open string instantons. Recall that the Chern-Simons perturbation expansion has been interpreted as a sum over virtual instantons at infinity in [40] (section 4.2.) These are essentially open string Riemann surfaces which degenerate to trivalent graphs geodesically embedded in $L$. In the present context, we can have a partial degeneration of a surface $\Sigma_{g, h}$ to another surface $\Sigma_{g^{\prime}, h^{\prime}}$ mapped to the disc $D$ and a trivalent graph with external legs ending on the boundary of $D$. For example, the degeneration of a sphere with three holes is represented in Fig.4. In the field theory limit, the sum over degenerate instantons is equivalent to a sum over trivalent graphs (in double line notation) with external legs ending on $\Gamma$. This is nothing else but the perturbative expansion of the Wilson line vacuum expectation values in Chern Simons theory performed above.

\section{The Geometry of Open String Maps}

We have shown so far that the open string instanton computations are in very good agreement with the predictions of large $N$ duality provided that one sums only over multicovers of a rigid disc $D$ in $Y$. A legitimate question at this point is if there are additional contributions from other components of the moduli space of 


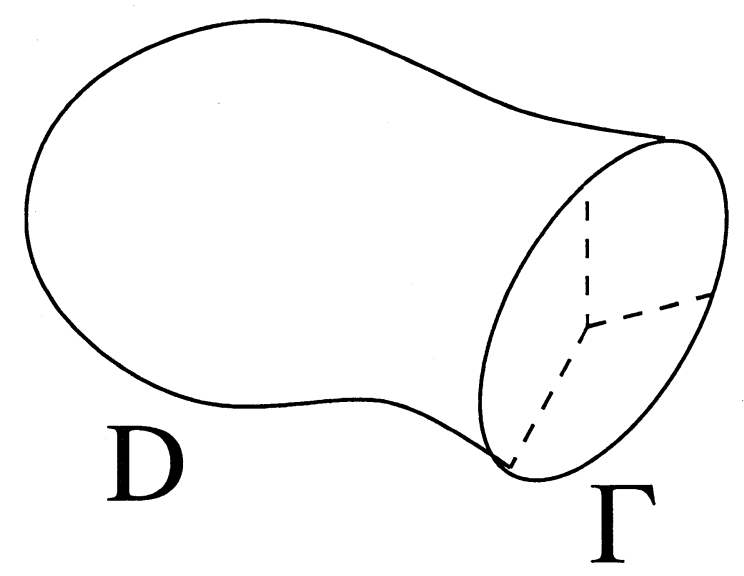

Figure 4: A degenerate open string instanton of type $(g, h)=(0,3)$

open string maps which may spoil this agreement. This is the problem we would like to address in this section, by developing a more formal approach to open string morphisms to the pair $(Y, L)$.

The main difficulty in answering this question is the lack of explicit constructions for generic open string maps in this background. This results in a very poor understanding of the moduli space $M_{g, h}(Y, L ; d[D])$ and its compactification. We can considerably simplify our task by adopting the strategy of [16, 23], which means we can restrict our considerations to open string maps invariant under a certain torus action on $Y$ which preserves $L$. In principle, this should allow us to settle the issue of extra contributions without a detailed knowledge of the moduli space.

In order to gain a better control on the geometry, we first compactify the hypersurfaces $Y_{\mu}$ by taking a projective closure of the ambient variety $Z$. Recall that $Z$ is isomorphic to the total space of $\mathcal{O}(-1) \oplus 2 \mathcal{O}$ over $\mathbb{P}^{1}$, which can be represented as a toric variety

$$
\begin{array}{llllll} 
& Z_{1} & Z_{2} & X & Y & V \\
\mathbb{C}^{*} & 1 & 1 & 0 & -1 & 0
\end{array}
$$

with disallowed locus $\left\{Z_{1}=Z_{2}=0\right\}$. The family $\mathcal{Y} / \Delta$ can be described as a family of hypersurfaces in $Z$ determined by

$$
Z_{1} V+X Y Z_{2}^{2}=\mu Z_{2}
$$

The local coordinates $(x, y, v, \lambda)$ and $(x, u, v, \rho)$ are standard affine toric coordinates for (35). For example, $\lambda=Z_{1} / Z_{2}, x=X, y=Y Z_{2}, v=V$. The (relative) 
projective closure of $Z$ is the compact toric variety $\bar{Z}=\left(\mathbb{C}^{6} \backslash F\right) /\left(\mathbb{C}^{*}\right)^{2}$ determined by

$\begin{array}{lcccccc} & Z_{1} & Z_{2} & X & Y & V & W \\ \mathbb{C}^{*} & 1 & 1 & 0 & -1 & 0 & 0 \\ \mathbb{C}^{*} & 0 & 0 & 1 & 1 & 1 & 1\end{array}$

with disallowed locus $F=\left\{Z_{1}=Z_{2}=0\right\} \cup\{X=Y=V=W=0\}$. Note that $\bar{Z} \simeq \mathbb{P}(3 \mathcal{O} \oplus \mathcal{O}(-1))$ over $\mathbb{P}^{1}$. Then the projective completion of the family (36) is a family $\overline{\mathcal{Y}} / \Delta$ of compact hypersurfaces in $\bar{Z}$ given by

$$
Z_{1} V W+X Y Z_{2}^{2}=\mu Z_{2} W^{2} .
$$

Let $\bar{Y}$ denote a generic fiber of this family (as noted before, we drop the subscript $\mu$ with the understanding that $\mu$ is fixed at some real positive value.) We denote by $Y \stackrel{i}{\hookrightarrow} \bar{Y} \stackrel{j}{\hookrightarrow} \bar{Z}$ the obvious embedding maps. The divisor at infinity on $\bar{Y}, \zeta_{\infty}$ is defined as the pull back of the Cartier divisor $W=0$ on $\bar{Z}$, so that $Y=\bar{Y} \backslash \zeta_{\infty}$. There is a subtlety related to this compactification, namely the variety $\bar{Y}$ is singular along the locus

$$
\left\{Z_{2}=V=W=0\right\} \cup\left\{Z_{1}=X=Y=W=0\right\}
$$

which is entirely contained in the divisor at infinity. In principle, one should blowup these singularities in order to have a good control over the geometry. However, for the present application this step is not really necessary since we can reduce our problem to questions about curves on $\bar{Z}$, which is smooth. After completing the argument, it will become clear that blowing up $\bar{Y}$ along the singular locus at infinity does not affect the conclusion.

For future reference, note that in terms of homogeneous coordinates the local coordinates on $Y$ are given by

$$
\begin{aligned}
& U_{1}: \quad \lambda=\frac{Z_{1}}{Z_{2}}, \quad x=\frac{X}{W}, \quad y=\frac{Y Z_{2}}{W}, \quad v=\frac{V}{W} \\
& U_{2}: \quad \rho=\frac{Z_{2}}{Z_{1}}, \quad x=\frac{X}{W}, \quad u=\frac{Y Z_{1}}{W}, \quad v=\frac{V}{W} .
\end{aligned}
$$

In the following, we will also need local coordinates at infinity defined in the open set $U_{3}=\left\{Z_{2} \neq 0, V \neq 0\right\}$

$$
\lambda=\frac{Z_{1}}{Z_{2}}, \quad x^{\prime}=\frac{X}{V}, \quad y^{\prime}=\frac{Y Z_{2}}{V}, \quad v^{\prime}=\frac{W}{V} .
$$

Let us record some details on the geometry of $\bar{Z}$. Since $\bar{Z}$ is toric and given by (37), its Picard group has rank two and it is generated by toric divisors. We can 
pick a system of generators $\left(\xi_{1}, \xi_{2}\right)$ determined by the divisor classes

$$
\xi_{1}:\left(Z_{1}=0\right)=\left(Z_{2}=0\right), \quad \xi_{2}:(X=0)=(V=0)=(W=0) .
$$

Note that $Y=0$ defines a divisor in the class $\xi_{2}-\xi_{1}$ and, using an explicit set of generators of the fan, we obtain the relations

$$
\begin{aligned}
& \xi_{1}^{2}=0, \quad \xi_{2}^{3}\left(\xi_{2}-\xi_{1}\right)=0 \\
& \xi_{1} \xi_{2}^{3}=1, \xi_{2}^{4}=1 .
\end{aligned}
$$

If we regard $\bar{Z}$ as a $\mathbb{P}^{3}$ fibration over $\mathbb{P}^{1}, \xi_{1}$ is the class of a fiber, and $\xi_{2}$ is the class of a relative hyperplane section. The Mori cone of $\bar{Z}$ is generated by curve classes corresponding to 3 -cones in the toric fan determined by the data (37). From (43) it is clear that a basis of the Mori cone is given by

$$
\eta_{1}=\xi_{2}^{2}\left(\xi_{2}-\xi_{1}\right), \quad \eta_{2}=\xi_{1} \xi_{2}^{2}
$$

Let us choose some convenient representatives of these curve classes

$$
\eta_{1}: X=Y=V=0, \quad \eta_{2}: Z_{1}=X=Y=0 .
$$

After these preliminary remarks, we are ready to discuss open string maps. As mentioned in the first paragraph of this section, we restrict our considerations to open string morphisms which are fixed points of a certain torus action. In the present context, we will consider a torus action on $Y$ induced by an action on $\bar{Y}$ which preserves the divisor at infinity. There is a natural $\left(S^{1}\right)^{4}$ action on $\bar{Z}$ with weights

$$
\begin{array}{cccccc}
Z_{1} & Z_{2} & X & Y & V & W \\
\lambda_{1} & 0 & \lambda_{2} & \lambda_{3} & \lambda_{4} & 0
\end{array}
$$

The subgroup $\left(S^{1}\right)^{2} \subset\left(S^{1}\right)^{4}$ defined by $\lambda_{1}+\lambda_{4}=0$ and $\lambda_{2}+\lambda_{3}=0$ preserves the hypersurface $\bar{Y}$ and the sphere $L$. It is also clear that the divisor $\zeta_{\infty}$ is invariant, therefore we obtain a well defined $\left(S^{1}\right)^{2}$-action on $Y$. For localization purposes, it suffices to consider a diagonal subgroup $T \subset\left(S^{1}\right)^{2}$ acting on $(Y, L)$. In the coordinate patch $U_{1}$, this action reads

$$
\lambda \rightarrow e^{-i \lambda_{1} \theta} \lambda, \quad x \rightarrow e^{-i \lambda_{2} \theta} x \quad y \rightarrow e^{i \lambda_{2} \theta} y \quad v \rightarrow e^{i \lambda_{1} \theta} v .
$$

Since the action of $T$ preserves $L$, it induces an action on the moduli space of open string morphisms with lagrangian boundary conditions on $L$. Our strategy is to find the fixed points of this action subject to a homology constraint. First note that any $T$-invariant stable map $f: \Sigma_{g, h} \rightarrow Y$ with $f\left(\partial \Sigma_{0,1}\right) \subset L$ must have the following form [16, 23, 30]. The domain $\Sigma_{g, h}$ is of the form $\Sigma_{g}^{0} \cup \Delta_{1} \cup \Delta_{2} \cup \ldots \Delta_{h}$ 
where $\Sigma_{g}^{0}$ is a prestable curve of arithmetic genus $g$ with $h$ marked points $p_{1}, \ldots p_{h}$ and $\Delta_{1}, \ldots, \Delta_{h}$ are discs attached to $\Sigma_{g}^{0}$ by identifying the origin of each disc with a point $p_{\alpha}, \alpha=1, \ldots, h$. The map $f_{\mid \Sigma_{g}^{0}}: \Sigma_{g}^{0} \rightarrow Y$ must be a $T$-invariant stable map to $Y$, and $f_{\mid \Delta_{\alpha}}: \Delta_{\alpha} \rightarrow Y$ must be a $T$-invariant map to a disc in $Y$ (with boundary) on $L$.

There is however a significant difference between our model and those of [16, $23,30]$. In those cases, although the target space $Y$ is a noncompact Calabi-Yau threefold, the maps actually take values in a compact submanifold thereof, which is simply a disc in $[23,30]$ and a projective plane in [16]. In our case we have certain components of the moduli space which consist of multicovers of a rigid disc, but we do not know a priori that these are all the components. Therefore we cannot a priori assume that the map $f: \Sigma_{g, h} \rightarrow Y$ takes values in a compact submanifold of $Y$. The correct treatment of this situation is to work in a relative setting, namely we should consider open string maps to the pair $(\bar{Y}, L)$ with prescribed order of contact along the divisor at infinity $\zeta_{\infty}$. For this, one should blow-up the singularities of $\bar{Y}$ at infinity, and consider maps to the resulting smooth three-fold. This would be an open string version of relative Gromov-Witten theory which will not be pursued here in detail. In principle, in this physical situation, one should consider open string morphisms with order of contact zero at infinity, and there would be very subtle questions related to the compactification of the moduli space. It will eventually become clear that for the present purposes we do not need to develop a full theory along these lines; it suffices to extend our search to $T$-invariant open string maps to the pair $(\bar{Y}, L)$ subject to the homology constraint $f_{*}\left[\Sigma_{g, h}\right]=d[D]$. Since $\bar{Y}$ has singularities at infinity, conceptually, we can think of maps $f: \Sigma_{g, h} \rightarrow \bar{Y}$ as maps $f: \Sigma_{g, h} \rightarrow \bar{Z}$ with boundary conditions on $L$, such that ${ }^{5} f_{*}\left[\Sigma_{g, h}\right]=j_{*} d[D]$ and the image of $f$ lies in $\bar{Y}$. This is the point of view we will take below, when we refer to open string maps to $\bar{Y}$. In the end, we will show that the structure of the fixed locus is such that there are no other contributions to the open string invariants besides those considered in the previous section.

Given the special structure of $T$-invariant open string maps, the problem reduces to finding $T$-invariant maps $f: \Sigma_{0,1} \rightarrow \bar{Y}$ with lagrangian boundary conditions along $L$. We claim that any such map can be extended to a $T$-invariant map $\bar{f}: \bar{\Sigma}_{0} \rightarrow \bar{Y}$ from a smooth genus zero curve $\bar{\Sigma}_{0} \simeq \mathbb{P}^{1}$ to $\bar{Y}$. This further reduces the problem to searching for $T$-invariant rational curves on $\bar{Y}$ which intersect $L$.

In order to justify this claim, recall that $L$ is defined in the coordinate patch

\footnotetext{
${ }^{5}$ Recall that $j: \bar{Y} \rightarrow \bar{Z}$ denotes the embedding. There is a subtlety related to this point, since we may have different homology classes in $H_{2}(\bar{Y}, L ; \mathbb{Z})$ which are mapped to the same homology class in $H_{2}(\bar{Z}, L, \mathbb{Z})$. This can be taken into account by a careful treatment.
} 
$(x, y, v, \lambda)$ by

$$
v \lambda+x y=\mu, \quad \lambda=\bar{v}, \quad x=\bar{y} .
$$

Take a tubular neighborhood $Q_{\epsilon}$ of $L$ in $Y$ (hence also in $\bar{Y}$ ) of the form $Q_{\epsilon}=$ $Y \cap B_{\epsilon}^{8}$, where $B_{\epsilon}^{8}$ is the eight-ball

$$
|\lambda|^{2}+|v|^{2}+|x|^{2}+|y|^{2} \leq 2\left(\mu^{2}+\epsilon^{2}\right)^{1 / 2}
$$

where $\epsilon \in \mathbb{R}_{+}$(recall that $\mu$ is also taken real and positive throughout this paper.) According to [8], $Q_{\epsilon}$ is a complex manifold with boundary diffeomorphic to $B^{3} \times S^{3}$. The boundary $S=\partial Q_{\epsilon}$ is diffeomorphic to $S^{2} \times S^{3}$. Moreover, since $L$ is lagrangian, it follows that $S$ is a contact hypersurface with respect to the symplectic form induced from $Y[10,11]$. If we take $\epsilon$ small enough, for any map $f: \Sigma_{0,1} \rightarrow \bar{Y}$ with $f\left(\partial \Sigma_{0,1}\right) \subset L, f\left(\Sigma_{0,1}\right) \cap Q_{\epsilon}$ is a small cylinder $\Xi$ embedded in $Q_{\epsilon}$ with the two boundary components mapped to $L$ and respectively $S$. Given the local form of the $T$-action (47), if $f: \Sigma_{0,1} \rightarrow \bar{Y}$ is $T$-invariant, there are only four such cylinders that can occur this way. We have

$$
\begin{aligned}
& \Xi_{1}: \mu^{1 / 2} \leq|\lambda| \leq\left[\left(\mu^{2}+\epsilon^{2}\right)^{1 / 2}+\epsilon\right]^{1 / 2}, v=\frac{\mu}{\lambda}, x=y=0 \\
& \Xi_{2}:\left[\left(\mu^{2}+\epsilon^{2}\right)^{1 / 2}-\epsilon\right]^{1 / 2} \leq|\lambda| \leq \mu^{1 / 2}, v=\frac{\mu}{\lambda}, x=y=0 \\
& \Xi_{3}: \mu^{1 / 2} \leq|x| \leq\left[\left(\mu^{2}+\epsilon^{2}\right)^{1 / 2}+\epsilon\right]^{1 / 2}, y=\frac{\mu}{x}, \lambda=v=0 \\
& \Xi_{4}:\left[\left(\mu^{2}+\epsilon^{2}\right)^{1 / 2}-\epsilon\right]^{1 / 2} \leq|x| \leq \mu^{1 / 2}, y=\frac{\mu}{x}, \lambda=v=0 .
\end{aligned}
$$

In each of these cases, we can find a suitable extension of $f$ to a map $\bar{f}: \bar{\Sigma}_{0} \rightarrow$ $\bar{Y}$. For example let us consider $\Xi_{1}$. Since $f$ is $T$-invariant, we can find a local coordinate $t$ on $f^{-1}\left(\Xi_{1}\right)$ and a positive integer $k$ such that $f$ is locally given by

$$
\lambda(t)=t^{k}, \quad v(t)=\frac{\mu}{t^{k}}, \quad x(t)=y(t)=0 .
$$

Note that in this parameterization, $f^{-1}\left(\Xi_{1}\right)$ is isomorphic to the annulus

$$
\mu^{1 / 2 k} \leq|t| \leq\left[\left(\mu^{2}+\epsilon^{2}\right)^{1 / 2}+\epsilon\right]^{1 / 2 k} .
$$

Now we can define a map from the disc

$$
D(\epsilon, k): \quad 0 \leq|t| \leq\left[\left(\mu^{2}+\epsilon^{2}\right)^{1 / 2}+\epsilon\right]^{1 / 2 k}
$$


to $\bar{Y}$ which in the local coordinates at infinity (41) reads

$$
\lambda=t^{k}, \quad v^{\prime}(t)=\frac{1}{\mu} t^{k}, \quad x(t)=y(t)=0 .
$$

Here we are forced to work in the coordinate patch at infinity since $v \rightarrow \infty$ as $t \rightarrow 0$ in (51). In particular, the origin of the disc $D(\epsilon, k)$ is mapped to the point at infinity $P=[0,1,0,0,1,0]$. Then we can glue the disc $D(\epsilon, k)$ to $\Sigma_{0,1}$ along the annulus (52) obtaining a smooth rational curve $\bar{\Sigma}_{0}$ and the map $f: \Sigma_{0,1} \rightarrow \bar{Y}$ extends by (54) to a map $\bar{f}: \bar{\Sigma}_{0} \rightarrow \bar{Y}$. By $T$-invariance, the image of this map has to be a rational curve in $\bar{Y}$ preserved by $T$ and passing through the point at infinity $P$. The only curve on $\bar{Y}$ satisfying these conditions is

$$
C_{1}: \quad Z_{1} V=\mu W Z_{2}, \quad X=Y=0 .
$$

By writing (55) in local coordinates, it follows that $C_{1}$ intersects $L$ along a circle which divides it into two discs with boundary on $L$. One of them $D_{1}$ is the disc $D$ considered in the previous section, while the second one $D_{1}^{\prime}$ is a disc in $\bar{Y}$ with origin at $P$. The invariant map $f: \Sigma_{0,1} \rightarrow \bar{Y}$ is a $k: 1$ cover of $D$.

Similar considerations apply to the other three cases in (50). For the second case, we obtain again the curve (55), but the roles of $D_{1}$ and $D_{1}^{\prime}$ are reversed. We now obtain a $k: 1$ cover of the disc $D_{1}^{\prime}$. For the remaining cases, we find a $T$-invariant curve

$$
C_{2}: \quad X Y Z_{2}=\mu W^{2}, \quad Z_{1}=V=0
$$

which is divided by $L$ into two discs $D_{2}, D_{2}^{\prime}$ in $\bar{Y}$. By contrast with the previous situation, both $D_{2}$ and $D_{2}^{\prime}$ intersect the divisor at infinity at $Q=[0,1,0,1,0,0]$ and respectively $R=[0,1,1,0,0,0]$. Moreover, the invariant map $f: \Sigma_{0,1} \rightarrow \bar{Y}$ is a $k: 1$ cover of $D_{2}$ and respectively $D_{2}^{\prime}$. To summarize this discussion, we conclude that an invariant map from a disc to $\bar{Y}$ with boundary conditions on $L$ must be a multicover of one of the four discs $D_{1}, D_{1}^{\prime}, D_{2}, D_{2}^{\prime}$ found above. Note that except $D_{1}=D$, the other three discs have points at infinity, hence they are not contained in the noncompact hypersurface $Y$. For this reason, one might be tempted at this point to rule out all fixed points consisting of maps with components along $D_{1}^{\prime}, D_{2}$ or $D_{2}^{\prime}$, keeping only maps with components along $D$. However, since we do not really understand the structure of the moduli space, we should proceed with more caution here.

We will show next that the discs $D_{1}^{\prime}, D_{2}$ or $D_{2}^{\prime}$ are in fact ruled out by the homology constraint $f_{*}\left[\Sigma_{g, h}\right]=d[D]$. The idea is to push forward homology classes to $\bar{Z}$, which is smooth, and use the structure of the Mori cone. First, note that using a standard exact sequence argument, we have an isomorphism

$$
0 \rightarrow H_{2}(\bar{Z}, \mathbb{Z}) \stackrel{\alpha}{\rightarrow} H_{2}(\bar{Z}, L ; \mathbb{Z}) \rightarrow 0 .
$$


Therefore, it suffices to compute the homology classes $\alpha^{-1}\left[D_{1}\right], \ldots, \alpha^{-1}\left[D_{2}^{\prime}\right]$ in terms of the generators $\eta_{1}, \eta_{2}$ of the Mori cone defined in (45). This is not quite straightforward, since the discs are rigid, and one cannot measure their homology class by using intersection theory as in the case of holomorphic curves. Instead we have to use the following deformation argument. Suppose we deform the sphere $L$ in $\bar{Z}$ be changing the value of $\mu$ to $\mu^{\prime}<\mu$. In this paragraph we restore the subindex $\mu$ for $Y, L$ in order to keep track of the $\mu$-dependence. Then the disc $D_{1 \mu}$ also changes to $D_{1 \mu^{\prime}}$ which can be obtained from $D_{1 \mu}$ by gluing in a small cylinder, which is fillable in $\bar{Z}$. This shows that $\alpha^{-1}\left[D_{1 \mu}\right]=\alpha^{-1}\left[D_{1 \mu^{\prime}}\right]$ for any $\mu, \mu^{\prime}$, and the same is true for the other three discs.

The advantage of this approach is that we can deform to $\mu=0$, such that the sphere $L_{\mu}$ shrinks to zero size. In this limit, the discs become holomorphic curves on $\bar{Z}$ whose homology classes in $H_{2}(\bar{Z}, \mathbb{Z})$ can be easily determined from the algebraic equations. Let us consider for example the discs $D_{1}, D_{1}^{\prime}$. In the limit $\mu=0$, the defining equations (55) of $C_{1}$ specialize to

$$
Z_{1} V=0, \quad X=Y=0 .
$$

Therefore $C_{1}$ specializes to a reducible curve with components $X=Y=V=0$ and respectively $Z_{1}=X=Y=0$, which are precisely the generators $(45)$ of $H_{2}(\bar{Z}, \mathbb{Z})$. The two discs $D_{1}, D_{1}^{\prime}$ are deformed in this limit to these two components of $C_{1}$, therefore we find

$$
\left[D_{1}\right]=\eta_{1}, \quad\left[D_{1}^{\prime}\right]=\eta_{2} .
$$

By a similar reasoning we also find $\left[D_{2}\right]=\left[D_{2}^{\prime}\right]=\eta_{2}$. Note that this deformation argument shows that the symplectic area of the disc $D$ is the same as that of the curve $C_{t}$ after transition. Therefore at classical level, $t=t_{o p}$ as noted at the end of section two.

Now we can determine the general structure of a $T$-invariant open string morphisms subject to the constraint $f_{*}\left[\Sigma_{g, h}\right]=d[D]$, with $d$ a positive integer. Since $\eta_{1}, \eta_{2}$ are generators of the Mori cone, it follows that for any such fixed point $\left.f\right|_{\Delta_{\alpha}}: \Delta_{\alpha} \rightarrow \bar{Y}$ must be a multicover of $D$. The other discs are indeed ruled out by homology constraints since one cannot have effective curves $C$ on $\bar{Z}$ so that $d \eta_{1}=[C]+d^{\prime} \eta_{2}$ for $d, d^{\prime}>0$. Moreover, by the same argument, the closed curve $\Sigma_{g}^{0}$ is mapped either to a point or to a $T$-invariant rational curve in the class $\eta_{1}$. Now, one can check that the only $T$-invariant curves on $\bar{Y}$ in this class are the sections defined by $X=Y=W=0, X=V=W=0$ and $V=Y=W=0$. These are all included in the divisor at infinity $\zeta_{\infty}$, and they are disconnected from the invariant disc $D$. Since the image of any map has to be connected, it follows that we cannot have fixed open string maps with components along the above curves. This leaves only maps that contract the curve $\Sigma_{g}^{0}$ to a point, while mapping $\Delta_{\alpha}$ 
to $D$. These are precisely the fixed points in the multicover moduli space of $D$, considered in $[23,30]$, whose contributions have been taken into account in the previous section. After this rather lengthy analysis, we can conclude that this is the complete answer.

\section{Acknowledgements:}

We are very grateful to Mina Aganagic and Cumrun Vafa for illuminating discussions and suggestions and to Ron Donagi and Tony Pantev for collaboration on a related project. We would also like to thank Bobby Acharya, Michael Douglas, John Etnyre, Albrecht Klemm, Andrew Kresch, Marcos Mariño, John McGreevy, Harald Skarke and Lisa Traynor for very stimulating conversations. We owe special thanks (and lots of tiramisù) to Corina Florea for invaluable help with the LaTeX conversion of the original draft. The work of D.-E. D. has been supported by DOE grant DOE-DE-FG02-96ER40959; A.G. is supported in part by the NSF Grant DMS-0074980.

\section{References}

[1] B. Acharya, M. Aganagic, K. Hori and C. Vafa, "Orientifolds, Mirror Symmetry and Superpotentials", hep-th/0202208.

[2] M. Aganagic and C. Vafa, "Mirror Symmetry, D-Branes and Counting Holomorphic Discs", hep-th/0012041.

[3] M. Aganagic, A. Klemm and C. Vafa, "Disk Instantons, Mirror Symmetry and the Duality Web", Z. Naturforsch. A 57 (2002) 1, hep-th/0105045.

[4] M. Aganagic and C. Vafa, "Mirror Symmetry and a $G_{2}$ Flop", hepth/0105225.

[5] M. Aganagic and C. Vafa, " $G_{2}$ Manifolds, Mirror Symmetry and Geometric Engineering", hep-th/0110171.

[6] J. Blum, "Calculation of Nonperturbative Terms in Open String Models", hep-th/0112039.

[7] T.-M. Chiang, A. Klemm, S.-T. Yau and E. Zaslow, "Local Mirror Symmetry: Calculations and Interpretations", ATMP 3 (1999) 495, hepth/9903053.

[8] H. Clemens, "Double Solids", Adv. Math. 47 (1983) 107. 
[9] M.R. Douglas, "Chern-Simons-Witten Theory as a Topological Fermi Liquid", hep-th/9403119.

[10] Y. Eliashberg, A. Givental and H. Hofer, "Introduction to Symplectic Field Theory", math.SG/0010059.

[11] J.B. Etnyre, "Symplectic Convexity in Low Dimensional Topology", Topology Appl. 88 (1998) 3.

[12] R. Gopakumar and C. Vafa, "Topological Gravity as Large $N$ Topological Gauge Theory", ATMP 2 (1998) 413, hep-th/9802016.

[13] R. Gopakumar and C. Vafa, "M-Theory and Topological Strings-I", hepth/9809187; "M-Theory and Topological Strings-II", hep-th/9812127.

[14] R. Gopakumar and C. Vafa, "On the Gauge Theory/Geometry Correspondence", ATMP 3 (1999) 1415, hep-th/9811131.

[15] S. Govindarajan, T. Jayaraman and T. Sarkar, "Disc Instantons in Linear Sigma Models", hep-th/0108234.

[16] T. Graber and E. Zaslow, "Open-String Gromov-Witten Invariants: Calculations and a Mirror Theorem" ", hep-th/0109075.

[17] M. Gross, "Topological Mirror Symmetry", math.AG/9909015.

[18] K. Hori and C. Vafa, "Mirror Symmetry", hep-th/0002222.

[19] K. Hori, A. Iqbal and C. Vafa, "D-Branes and Mirror Symmetry", hepth/0005247.

[20] A. Iqbal and A.-K. Kashani-Poor, "Discrete Symmetries of the Superpotential and Calculation of Disk Invariants", hep-th/0109214.

[21] S. Kachru, S. Katz, A. Lawrence and J. McGreevy, "Open String Instantons and Superpotentials", Phys. Rev. D62 (2000) 026001, hepth/9912151.

[22] S. Kachru, S. Katz, A. Lawrence and J. McGreevy, "Mirror Symmetry for Open Strings", hep-th/0006047.

[23] S. Katz and C.-C. M. Liu, "Enumerative Geometry of Stable Maps with Lagrangian Boundary Conditions and Multiple Covers of the Disc", ATMP 5 (2001) 1, math.AG/0103074.

[24] A. Klemm, S. Katz and C. Vafa, "M-Theory, Topological Strings and Spinning Black Holes", ATMP 3 (1999) 1445, hep-th/9910181. 
[25] J.M.F. Labastida and M. Mariño, "Polynomial Invariants for Torus Knots and Topological Strings", Commun. Math. Phys. 217 (2001) 423, hepth/0004196.

[26] J.M.F. Labastida, M. Mariño and C. Vafa, "Knots, Links and Branes at Large N", JHEP 11 (2000) 007, hep-th/0010102.

[27] J.M.F. Labastida and M. Mariño, "A New Point of View in the Theory of Knot and Link Invariants", math.QA/0104180.

[28] W. Lerche and P. Mayr, "On $\mathcal{N}=1$ Mirror Symmetry for Open Type II Strings", Hep-th/0111113.

[29] N.-C. Leung and C. Vafa, "Branes and Toric Geometry", ATMP 2 (1998) 91, hep-th/9711013.

[30] J. Li and Y.S. Song, "Open String Instantons and Relative Stable Morphisms", hep-th/0103100.

[31] M. Mariño and G. Moore, "Counting higher genus curves in a Calabi-Yau manifold",Nucl.Phys. B543 (1999) 592, hep-th/9808131.

[32] M. Mariño and C. Vafa, "Framed Knots at Large N", hep-th/0108064.

[33] P. Mayr, "N $=1$ Mirror Symmetry and Open/Closed String Duality", hep-th/0108229.

[34] P. Mayr, "Summing Up Open String Instantons and $\mathcal{N}=1$ String Amplitudes", hep-th/0203237.

[35] H. Ooguri and C. Vafa, "Knot Invariants and Topological Strings", Nucl. Phys. B 577 (2000) 419, hep-th/9912123.

[36] V. Periwal, "Topological Closed String Interpretation of Chern-Simons Theory", Phys. Rev. Lett. 71 (1993) 125, hep-th/9305115.

[37] P. Ramadevi and T. Sarkar, "On Link Invariants and Topological String Amplitudes", Nucl. Phys. B600 (2001) 487, hep-th/0009188.

[38] C. Vafa, "Extending Mirror Conjecture to Calabi-Yau with Bundles", hep-th/9804131.

[39] C. Vafa, "Superstrings and Topological Strings at Large N", J. Math. Phys. 42 (2001) 2798, hep-th/0008142.

[40] E. Witten, "Chern-Simons Gauge Theory as a String Theory", "The Floer Memorial Volume", H. Hofer, C.H. Taubes, A. Weinstein and E. Zehnder, eds, Birkhäuser 1995, 637, hep-th/9207094. 